\title{
Simple presurgical method of predicting C5 palsy after cervical laminoplasty using $\mathrm{C} 5$ nerve root ultrasonography
}

\author{
Mikinobu Takeuchi, MD, PhD, ${ }^{1,2,5}$ Norimitsu Wakao, MD, PhD, ${ }^{1,3}$ Mitsuhiro Kamiya, MD, PhD, ${ }^{1,3}$ \\ Atsuhiko Hirasawa, MD, ${ }^{1,3}$ Kenta Murotani, PhD, ${ }^{4}$ and Masakazu Takayasu, MD, PhD',2 \\ ${ }^{1}$ Spine Center, ${ }^{2}$ Department of Neurological Surgery, ${ }^{3}$ Department of Orthopedic Surgery, and ${ }^{4}$ Biostatistics and Clinical \\ Research Center, Aichi Medical University Hospital, Nagakute, Aichi, Japan, and ${ }^{5}$ Department of Spine Surgery, Aichi Spine \\ Hospital, Inuyama, Aichi, Japan
}

\begin{abstract}
OBJECTIVE The incidence of C5 palsy after cervical laminoplasty is approximately $5 \%$. Because C5 palsy is related to cervical foraminal stenosis at the C4-5 level, the authors hypothesized that cervical foraminal stenosis can be diagnosed by examining the $\mathrm{C} 5$ nerve root (NR) using ultrasonography. The purpose of this study was to investigate whether postoperative $\mathrm{C} 5$ palsy could be predicted using ultrasonography.

METHODS This study used a prospective diagnosis design. In total, 140 patients undergoing cervical laminoplasty were examined with ultrasound. The cross-sectional area (CSA) of the C5 NR was measured on both sides before surgery, and the incidence of postoperative C5 palsy was examined. The difference between the CSA of the patients with and without C5 palsy and the lateral differences in the C5 palsy group were determined.

RESULTS The incidence of C5 palsy was $5 \%$ (7 cases). Symptoms manifested at a median of 5 days after surgery (range 1-29 days). The CSA of the C5 NR on the affected side was significantly enlarged in the C5 palsy group compared with that in the no-C5 palsy group $(p=0.001)$. In addition, in the patients who had C5 palsy, the CSA of the C5 NR was significantly enlarged on the affected side compared with that on the unaffected side $(p=0.02)$. Receiver operating characteristic analysis indicated that the best threshold value for the CSA of the C5 NR was $10.4 \mathrm{~mm}^{2}$, which provided $91 \%$ sensitivity and $71 \%$ specificity.
\end{abstract}

CONCLUSIONS C5 palsy may be predicted preoperatively using ultrasound. The authors recommend the ultrasonographic measurement of the CSA of the C5 NR prior to cervical laminoplasty.

https://thejns.org/doi/abs/10.3171/2018.2.SPINE171363

KEYWORDS C5 nerve root; C5 palsy; foraminal stenosis; ultrasonography; cervical laminoplasty

$\mathrm{T}$ HE incidence of C5 palsy after cervical laminoplasty is approximately $5 \%$., ${ }^{3,6,10,14} \mathrm{C} 5$ palsy can be caused by cervical foraminal stenosis, ${ }^{8,9}$ reperfusion syndrome,${ }^{5}$ spinal cord shift, ${ }^{16}$ or heat injury. ${ }^{7}$ Particularly in cases of cervical foraminal stenosis, some reports have described presurgical predictive methods: thin-slice $\mathrm{CT}^{8}$ and various MRI methods (oblique MRI ${ }^{12}$ or MR myelography $^{2}$ ) to avoid C5 palsy after cervical laminoplasty. It is probable that cervical foraminal stenosis contributes to C5 palsy after cervical laminoplasty. However, the reliability of evaluations remains unclear because MRI and CT scans often show multilevel compression.

Some recent reports have indicated that cervical foraminal stenosis is related to an enlarged cervical nerve root (NR) at the same level on the affected side and that this enlarged cervical NR might be detectable with ultrasonography. ${ }^{11,19}$ Moreover, 3D MRI has revealed that at the lumbar level, NRs entrapped by foraminal stenosis become swollen. ${ }^{4}$

We hypothesized that C5 palsy could be predicted by

ABBREVIATIONS CSA = cross-sectional area; $\mathrm{CSM}=$ cervical spondylotic myelopathy; $\mathrm{NR}=$ nerve root; JOA = Japanese Orthopaedic Association; MMT = manual muscle test; OPLL = ossification of the posterior longitudinal ligament; ROC = receiver operating characteristic; $T P=$ transverse process.

SUBMITTED December 8, 2017. ACCEPTED February 14, 2018.

INCLUDE WHEN CITING Published online July 6, 2018; DOI: 10.3171/2018.2.SPINE171363. 
examining the C5 NR using cervical ultrasonography before cervical laminoplasty.

\section{Methods}

The institutional review board of Aichi Medical University Hospital approved this study, and informed consent was obtained from all participating patients. This prospective cohort study was performed between December 2014 and April 2017 at our hospital and included patients with cervical spondylotic myelopathy (CSM).

\section{Inclusion and Exclusion Criteria}

First, the patients who were eligible for inclusion suffered from CSM symptoms in the arm, hand, or finger, including paresthesias, numbness and sensory changes, weakness, or abnormal deep tendon reflexes. Second, the patients were required to have undergone a cervical MRI that revealed cervical osteophytes, ossification of the posterior longitudinal ligament (OPLL), and cervical disc herniation that compressed the cervical spinal cord. Third, patients who were going to undergo cervical laminoplasty of at least the C4 and C5 levels were included. The surgical procedure used for the cervical laminoplasty, such as the open door or French door method, was selected by the spine surgeon.

Patients were excluded if they had experienced 1) cervical radiculopathy only; 2) neck pain only; 3) deltoid muscle weakness; 4) severe diabetes, renal, or liver dysfunction; 5) myopathy, neuropathy, or collagen diseases; 6) trauma; or 7) additional posterior cervical fusions. Among the 160 patients who were considered for enrollment in the study, 147 met the baseline inclusion criteria. However, an additional 7 patients were excluded because clear ultrasonography images could not be obtained before the cervical laminoplasty. In total, data from 140 patients were included in the analysis (Fig. 1). Postoperative C5 palsy was defined as the presence of deltoid muscle weakness of grade 3 or less, according to a manual muscle test (MMT) with or without biceps weakness.

\section{Surgical Procedure}

The open door or the French door laminoplasty method was performed with the patient in the prone position under general anesthesia. During exposure of the cervical spine, the spinal processes were split at the midline and cut at the base. In the open door method, a right-side gutter was made and used as a hinge at the lateral border of the laminae, and a left-side gutter on the contralateral side was produced as an open side. The open side of the lamina was lifted and fixed with hydroxyapatite spacers (HOYA Technosurgical Co. Ltd.) and nylon sutures. In the open door method, the laminae were split at the midline using a surgical drill. Bilateral gutters were created as hinges at the lateral border of the laminae. The treated laminae were elevated like a French door and then fixed with the hydroxyapatite spacers. Foraminotomy was not performed in all cases.

\section{Radiological Evaluation}

Cervical alignment of the $\mathrm{C} 2-\mathrm{C} 7 \mathrm{Cobb}$ angle was de- termined on preoperative standing lateral radiographs obtained in neutral, flexion, and extension positions. OPLL was diagnosed on CT scans. High T2 signal intensity at C3-4 or C4-5 was determined on the basis of preoperative MRI.

\section{Ultrasonography Measurements}

Four musculoskeletal ultrasound experts who each had more than 5 years of experience performed ultrasonography using a real-time scanner with a $13-5 \mathrm{MHz}$ linear array probe before cervical laminoplasty. These individuals were blinded to the patients' clinical characteristics or radiological findings.

For the ultrasound examination, the patients were placed in a lateral decubitus position..$^{18}$ The examiners set the ultrasound linear array probe to the brightness mode and stood behind the subject during the examination. They investigated the C5, C6, and C7 NRs bilaterally on an axial image by perceiving the cervical transverse process (TP) form. The C5 and C6 TPs have obvious anterior and posterior tubercles. In contrast, the C7 TP has an unremarkable anterior tubercle and a prominent posterior tubercle. Thus, the C7 TP is an important landmark. ${ }^{17}$

The examiners measured the cross-sectional area $\left(\mathrm{CSA}\right.$, in $\left.\mathrm{mm}^{2}\right)$ of the bilateral C5 NRs.

The C5 NR measurement point was standardized. The CSA of the C5 NR was measured where the examiners found the C5 NR between the anterior and posterior tubercles of the TP.

We assessed 4 components: 1) The affected CSAs of the C5 NR of the subjects with postoperative C5 palsy (C5 palsy group) were compared with those of the subjects in the group without C5 palsy (no-C5 palsy group). 2) The difference between the CSA of the affected C5 NR and the unaffected C5 NR was calculated in the C5 palsy patients. 3) The risk factors for C5 palsy were examined using multivariate logistic regression analyses. 4) The sensitivity, specificity, and cutoff values of the CSA of the C5 NR were investigated for the C5 palsy and no-C5 palsy groups.

\section{Sample Size}

Based on a previously published pilot study performed by our group,${ }^{17}$ we estimated that patients with symptomatic C5 NR foraminal stenosis would have a CSA of the C5 NR of $14.2 \pm 3.1 \mathrm{~mm}^{2}$ compared with a CSA of $6.4 \pm$ 1.7 for those without C5 NR foraminal stenosis. ${ }^{19}$ Assuming a 5\% postoperative $\mathrm{C} 5$ palsy rate, we determined that we needed a sample size of 140 patients (of which 7 were likely to develop C5 palsy) in order to achieve an alpha of 0.05 and a power of 0.80 .

\section{Statistical Analysis}

The baseline characteristics of various factors were compared between the C5 palsy and no-C5 palsy groups using t-tests or chi-square tests. Multivariate logistic regression analyses were used to explore the risk factors for C5 palsy after cervical laminoplasty. The receiver operating characteristic (ROC) curve was used to examine the sensitivity, specificity, and cutoff values of the CSA for the 


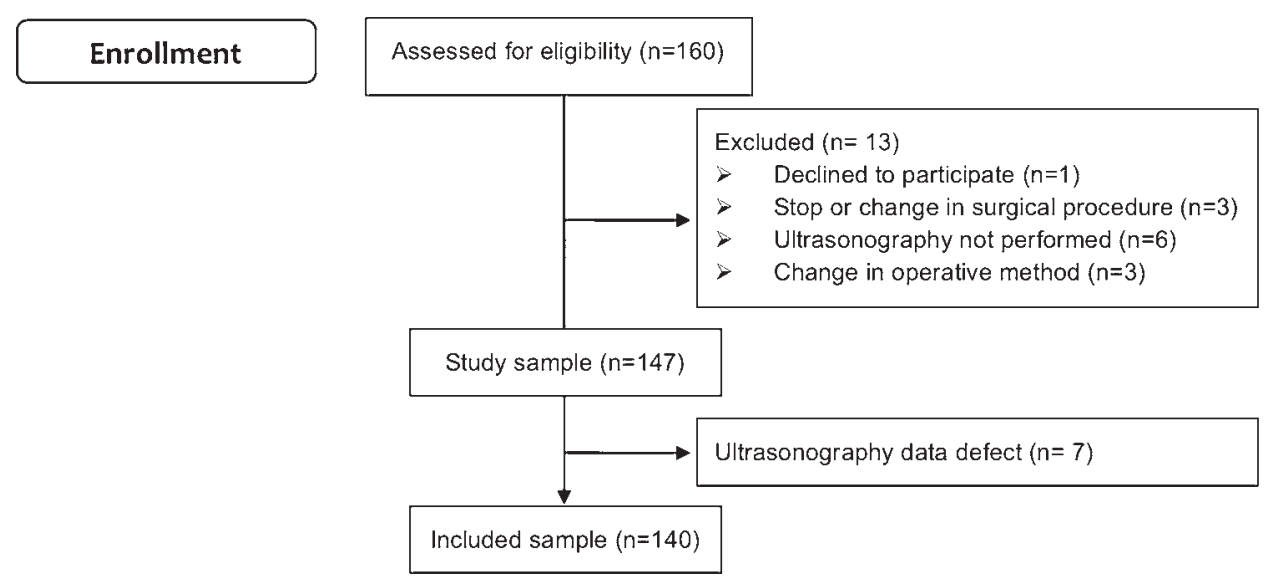

FIG. 1. Flow diagram showing patient selection for this study. In the 6 cases in which patients were excluded due to ultrasonography not being performed, the reasons were as follows: in 4 cases, the patients forgot the ultrasonography appointment after they had been enrolled in the study; in the remaining 2 cases, the ultrasonography examination was not performed due to a scheduling omission.

C5 NR. Intraclass correlation coefficients of ultrasound measurements for the 4 examiners were calculated based on examinations they each performed on 5 normal volunteers. Data were analyzed using SPSS software (version 22; IBM Corp.). The significance level was set to 0.05 .

\section{Results}

The incidence of C5 palsy was 5\% (7 of 140 patients). The timing of the initial manifestation of C5 palsy ranged from 1 to 29 days (median 5 days) after surgery. The baseline characteristics of the no-C5 palsy group (133 patients, including 89 men and 44 women; mean age 67 years) and the C5 palsy group (7 patients, including 4 men and 3 women; mean age 63 years) are presented in Table 1 . The 2 groups did not differ significantly in terms of age, symptom duration, sex, diabetes status, smoking status, Japanese Orthopaedic Association (JOA) score, radiographic assessments, MRI findings, or surgical methods (Table 1).

On the cervical ultrasound, the CSA of the C5 NR was significantly enlarged on the affected side in the C5 palsy group $\left(10.8 \pm 4.2 \mathrm{~mm}^{2}\right)$ compared with that in the no-C5 palsy group $\left(7.1 \pm 2.3 \mathrm{~mm}^{2}\right)(\mathrm{p}=0.001)$. However, there was no significant between-groups difference with respect to the CSA of the C5 NR on the unaffected side (Figs. 2 and 3).

TABLE 1. Baseline characteristics

\begin{tabular}{|c|c|c|c|c|}
\hline Characteristic & No C5 Palsy $(n=133)$ & C5 Palsy $(n=7)$ & Difference $(95 \% \mathrm{Cl})$ & $\mathrm{p}$ Value \\
\hline Mean age in yrs & $67 \pm 11$ & $63 \pm 14$ & $4.0(-4.5$ to 13$)$ & 0.3 \\
\hline Mean duration of CSM Sx in mos & $12 \pm 11$ & $12 \pm 9$ & $0.6(-9.3$ to 8.1$)$ & 0.9 \\
\hline Sex, $n$ & & & & 0.7 \\
\hline Male & 89 & 4 & & \\
\hline Female & 44 & 3 & & \\
\hline Diabetes, n (\%) & $34(26)$ & $1(14)$ & & 0.7 \\
\hline Smoking, $\mathrm{n}(\%)$ & $36(27)$ & $2(29)$ & & 0.1 \\
\hline Mean JOA score & $10.7 \pm 2.6$ & $11.2 \pm 2$ & $0.5(-2.5$ to 1.5$)$ & 0.6 \\
\hline \multicolumn{5}{|l|}{ Imaging findings } \\
\hline \multicolumn{5}{|l|}{ Cobb angle } \\
\hline Neutral & $10.5^{\circ} \pm 13.8^{\circ}$ & $12.3^{\circ} \pm 11.4^{\circ}$ & $1.7^{\circ}\left(-12.2^{\circ}\right.$ to $\left.8.7^{\circ}\right)$ & 0.7 \\
\hline Flexion & $-11.9^{\circ} \pm 15^{\circ}$ & $-8.9^{\circ} \pm 8.8^{\circ}$ & $3^{\circ}\left(-14.3^{\circ}\right.$ to $\left.8.3^{\circ}\right)$ & 0.6 \\
\hline Extension & $25^{\circ} \pm 36.5^{\circ}$ & $27.7^{\circ} \pm 10^{\circ}$ & $2.8^{\circ}\left(-30^{\circ}\right.$ to $\left.24.6^{\circ}\right)$ & 0.8 \\
\hline OPLL, n (\%) & $20(15)$ & $1(14)$ & & 1 \\
\hline High T2 signal intensity at C3-4 or C4-5, n (\%) & $37(28)$ & $2(29)$ & & 1 \\
\hline \multicolumn{5}{|l|}{ Surgical method, $\mathrm{n}$} \\
\hline Open door & 51 & 3 & & \\
\hline French door & 82 & 4 & & \\
\hline
\end{tabular}

Sx $=$ symptoms. 


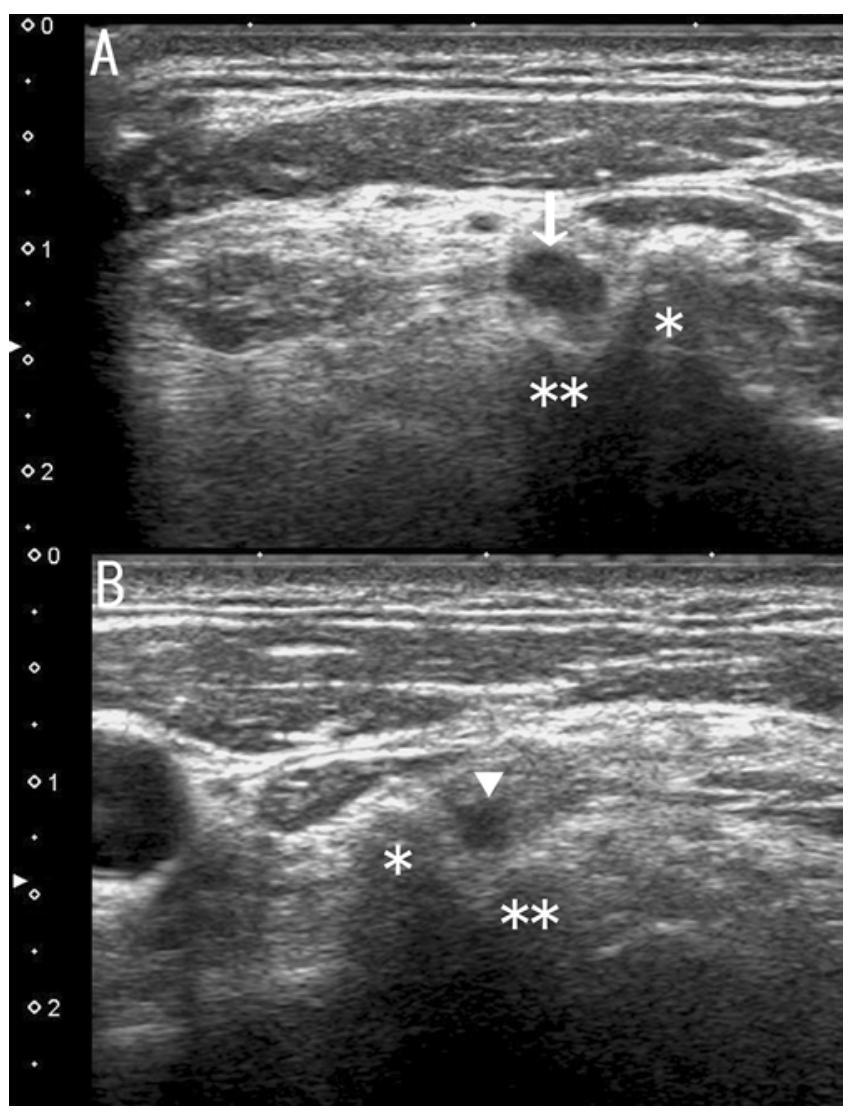

FIG. 2. C5 NR ultrasonography. A: The CSA of the C5 NR (arrow) in the C5 palsy group. B: The CSA of the C5 NR (arrowhead) in the no-C5 palsy group. The asterisks $\left({ }^{*}\right)$ indicate the anterior tubercle of the $\mathrm{C} 5$ transverse process. The double asterisks $\left.{ }^{* *}\right)$ indicate the posterior tubercle of the $\mathrm{C} 5$ transverse process.

In the C5 palsy group, the CSA of the C5 NR was significantly greater on the affected side $\left(10.8 \pm 4.2 \mathrm{~mm}^{2}\right)$ than on the unaffected side $\left(6.3 \pm 1.9 \mathrm{~mm}^{2}\right)(\mathrm{p}=0.02)$ (Figs. 2 and 3).

Multivariate logistic regression analyses of the $\mathrm{C} 5$ palsy group after cervical laminoplasty indicated that enlargement of the CSA of the C5 NR was a risk factor $(\mathrm{B}=$ 0.5, OR $1.6,95 \%$ CI $1.2-2.3, \mathrm{p}=0.005$ ) (Table 2).

Table 3 presents the threshold values of the CSA of the C5 NR obtained using ROC analysis. The best threshold value for the C5 NR was $10.4 \mathrm{~mm}^{2}$ (CSA), with $91 \%$ sensitivity and $71 \%$ specificity. The area under the curve for the CSA was 0.79 (95\% CI 0.59-0.98).

The interrater reliability of the CSA measurements was 0.71 (95\% CI 0.49-0.85). Moreover the intrarater reliability ratings of the 4 examiners were 0.71 (95\% CI $0.37-$ 0.87 ), 0.61 (95\% CI 0.1-0.83), 0.71 (95\% CI 0.33-0.87), and 0.74 (95\% CI 0.4-0.89).

\section{Discussion}

This prospective study investigated the CSA of the C5 NR using cervical ultrasonography. We hypothesized that enlargement of the C5 NR before cervical laminoplasty might result in C5 palsy. The investigation was

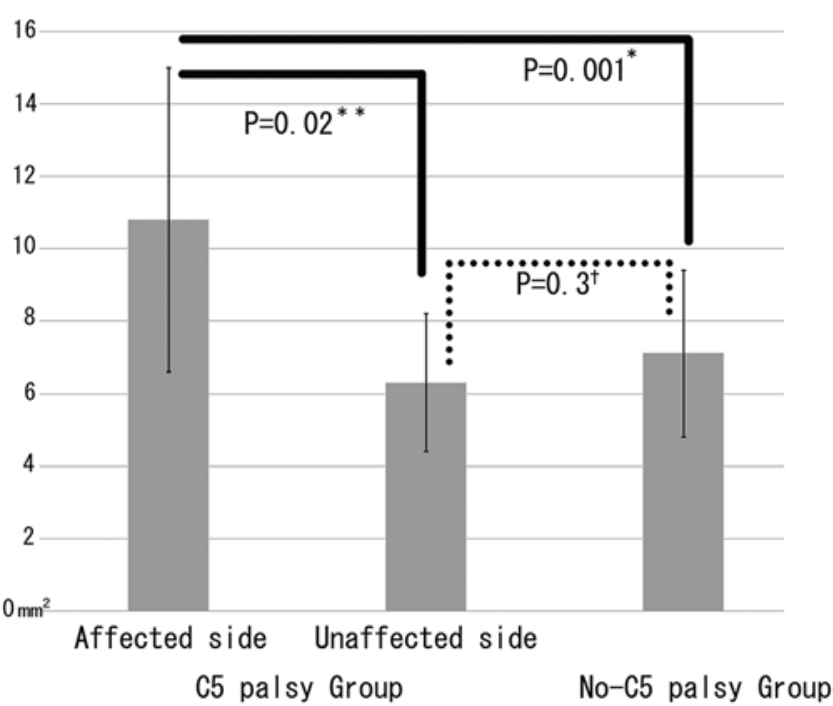

FIG. 3. The CSA of the C5 NR was compared between the C5 palsy and no-C5 palsy groups. ${ }^{*}$ Comparison of the CSA of the C 5 NR on the affected side $\left(10.8 \pm 4.2 \mathrm{~mm}^{2}\right)$ vs the unaffected side $\left(6.3 \pm 1.9 \mathrm{~mm}^{2}\right)$ in the C5 palsy group $(p=0.02) .{ }^{*}$ Comparison of the CSA of the C5 NR of the affected side of the C5 palsy group $\left(10.8 \pm 4.2 \mathrm{~mm}^{2}\right)$ vs the no-C5 palsy group $\left(7.1 \pm 2.3 \mathrm{~mm}^{2}\right)(p=0.001)$. †Comparison of the CSA of the C5 NR on the unaffected side in the C5 palsy group $\left(6.3 \pm 1.9 \mathrm{~mm}^{2}\right)$ vs the no-C5 palsy group $(7.1 \pm 2.3 \mathrm{~mm})(p=0.3)$.

accomplished by comparing the preoperative CSA of the C5 NR in patients who subsequently developed C5 palsy to measurements obtained in patients who did not develop C5 palsy. We obtained 3 important findings from this study. First, we determined that the threshold value of the C5 NR was $10.4 \mathrm{~mm}^{2}$ (CSA), with $91 \%$ sensitivity and $71 \%$ specificity. Second, the CSA of the affected C5 NR in the C5 palsy group was significantly larger than both the CSA of the C5 NR in the no-C5 palsy group and the CSA of the unaffected C5 NR in the C5 palsy group. Third, enlargement of the CSA of the C5 NR was confirmed as a risk factor for C5 palsy after cervical laminoplasty.

Previous reports have observed a relationship between cervical foraminal stenosis at the $\mathrm{C} 4-5$ level and $\mathrm{C} 5$ palsy after cervical laminoplasty. ${ }^{89}$ Recent articles have report-

TABLE 2. Multivariate logistic regression analyses of the risk factors for C5 palsy after cervical laminoplasty

\begin{tabular}{lcll}
\hline \multicolumn{1}{c}{ Variable } & B & OR $(95 \%$ Cl $)$ & p Value \\
\hline Sex & 0.4 & $1.4(0.2-9.9)$ & 0.7 \\
\hline Age & 0.01 & $0.9(0.9-1)$ & 0.1 \\
\hline Diabetes & -0.1 & $0.9(0.1-8.8)$ & 0.9 \\
\hline Smoking & -0.1 & $0.8(0.1-6.5)$ & 0.9 \\
\hline CSA of C5 NR & 0.5 & $1.6(1.2-2.3)$ & 0.005 \\
\hline OPLL & 0.6 & $1.8(0.1-22)$ & 0.6 \\
\hline $\begin{array}{l}\text { High T2 signal intensity at } \\
\text { C3-4 or C4-5 }\end{array}$ & -0.4 & $0.7(0.1-6.5)$ & 0.7 \\
\hline
\end{tabular}

$\mathrm{OR}=$ odds ratio. 
TABLE 3. Sensitivity and specificity of CSA for diagnosing C5 palsy

\begin{tabular}{lccccc}
\hline Threshold & Sensitivity & Specificity & LR+ & LR- & AUC $(95 \% \mathrm{Cl})$ \\
\hline $5.7 \mathrm{~mm}^{2}$ & $30 \%$ & $100 \%$ & & 0.7 & \\
\hline $7.7 \mathrm{~mm}^{2}$ & $41 \%$ & $86 \%$ & 2.9 & 0.7 & \\
\hline $10.4 \mathrm{~mm}^{2}$ & $91 \%$ & $71 \%$ & 3.1 & 0.1 & \\
\hline $14.2 \mathrm{~mm}^{2}$ & $99.2 \%$ & $14 \%$ & 1.2 & 0.1 & $0.79(0.59-0.98)$ \\
\hline
\end{tabular}

$\mathrm{AUC}=$ area under the curve; $\mathrm{LR}-=$ negative likelihood ratio; $L R+=$ positive likelihood ratio.

ed that cervical foraminal stenosis led to swelling of the cervical NR at the affected level that could be detected with ultrasonography. ${ }^{11,19}$ Thus, cervical laminoplasty for patients with a swollen C5 NR may result in postsurgical C5 palsy. It was clear that postsurgical C5 palsy occurred in the patients with a swollen C5 NR.

Cervical nerve enlargement is believed to be caused by a relationship between entrapment neuropathy and focal vascular impairment. A report has demonstrated that external entrapment of a peripheral nerve leads to changes of internal construction in entrapment diseases and that deteriorated venous perfusion leads to increased reversible intraneural edema and intraneural pressure. ${ }^{1}$ Another study has also demonstrated that increased permeability was exhibited within the compressed nerve root in entrapment neuropathies. The compressed nerve root leads to fibrosis and secondary chronic edema. ${ }^{15}$ Therefore, patients with cervical foraminal stenosis presented with an enlarged C5 NR because of C5 NR entrapment at the C4-5 level, and after cervical laminoplasty, the kink in the C5 NR became more severe in association with the posterior shift of the spinal cord, thereby leading to C5 palsy.

A systematic review article has reported the following as risk factors for postsurgical C5 palsy: advanced age, sex, OPLL, high T2 signal in the spinal cord, and cervical foraminal stenosis. ${ }^{3,6}$ In our study, an enlarged C5 NR indicative of cervical foraminal stenosis was the only identified risk factor. We consider our results to be compatible with those of various previous reports.

Our results show that if the laminoplasty was performed in the patients with CSM and an enlarged C5 NR, the risk of postsurgical C5 palsy was high. Thus, we recommend that in such cases the surgical procedure should be changed to anterior decompression and fusion or be performed in combination with posterior foraminotomy.

\section{Limitations}

This study has some limitations. First, cervical ultrasonographic examination is not currently in use worldwide. Nevertheless, ultrasonography is a simple examination that is widely performed for the thyroid, heart, liver, or abdominal regions, and we believe that cervical ultrasonography will be a popular examination in the future. Second, patients who underwent posterior decompression with fusion were excluded. Posterior fusion surgery increases the likelihood of C5 palsy (reported rate of 11\%) because of iatrogenic postsurgical foraminal stenosis due to the correction of cervical alignment. ${ }^{13}$ Because the preopera- tive CSA of the C5 NR may be normal in these cases, our technique would not be expected to be reliable for predicting postoperative $\mathrm{C} 5$ palsy in patients undergoing fusion surgery.

\section{Conclusions}

A diagnostic threshold with high sensitivity and specificity was identified for the CSA of the C5 NR associated with $\mathrm{C} 5$ palsy. We recommend ultrasonographic measurement of the CSA of the C5 NR before performing cervical laminoplasty.

\section{Acknowledgments}

We thank the following hospitals and ultrasound technicians for their cooperation with the study: Aichi Medical University (Mrs. Kumiko Kiba, Miss Yasuko Kuroda, Miss Keiko Hasegawa, and Mrs. Minako Tsukamoto), Shirayama Surgical Clinic (Mr. Masaya Kato), and Asai Hospital (Dr. Toshimasa Asai).

\section{References}

1. Bianchi S, Montet X, Martinoli C, Bonvin F, Fasel J: Highresolution sonography of compressive neuropathies of the wrist. J Clin Ultrasound 32:451-461, 2004

2. Birchall D, Connelly D, Walker L, Hall K: Evaluation of magnetic resonance myelography in the investigation of cervical spondylotic radiculopathy. Br J Radiol 76:525-531, 2003

3. Bydon M, Macki M, Kaloostian P, Sciubba DM, Wolinsky JP, Gokaslan ZL, et al: Incidence and prognostic factors of C5 palsy: a clinical study of 1001 cases and review of the literature. Neurosurgery 74:595-605, 2014

4. Byun WM, Jang HW, Kim SW: Three-dimensional magnetic resonance rendering imaging of lumbosacral radiculography in the diagnosis of symptomatic extraforaminal disc herniation with or without foraminal extension. Spine (Phila Pa 1976) 37:840-844, 2012

5. Chiba K, Toyama Y, Matsumoto M, Maruiwa H, Watanabe M, Hirabayashi K: Segmental motor paralysis after expansive open-door laminoplasty. Spine (Phila Pa 1976) 27:2108-2115, 2002

6. Gu Y, Cao P, Gao R, Tian Y, Liang L, Wang C, et al: Incidence and risk factors of C5 palsy following posterior cervical decompression: a systematic review. PLoS One 9:e101933, 2014

7. Hosono N, Miwa T, Mukai Y, Takenaka S, Makino T, Fuji T: Potential risk of thermal damage to cervical nerve roots by a high-speed drill. J Bone Joint Surg Br 91:1541-1544, 2009

8. Imagama S, Matsuyama Y, Yukawa Y, Kawakami N, Kamiya M, Kanemura T, et al: C5 palsy after cervical laminoplasty: a multicentre study. J Bone Joint Surg Br 92:393-400, 2010

9. Katsumi K, Yamazaki A, Watanabe K, Ohashi M, Shoji H: Analysis of C5 palsy after cervical open-door laminoplasty: relationship between C5 palsy and foraminal stenosis. J Spinal Disord Tech 26:177-182, 2013

10. Katsumi K, Yamazaki A, Watanabe K, Ohashi M, Shoji H: Can prophylactic bilateral C4/C5 foraminotomy prevent postoperative $\mathrm{C} 5$ palsy after open-door laminoplasty?: a prospective study. Spine (Phila Pa 1976) 37:748-754, 2012

11. Kim E, Yoon JS, Kang HJ: Ultrasonographic cross-sectional area of spinal nerve roots in cervical radiculopathy: a pilot study. Am J Phys Med Rehabil 94:159-164, 2015

12. Lubelski D, Derakhshan A, Nowacki AS, Wang JC, Steinmetz MP, Benzel EC, et al: Predicting C5 palsy via the use of preoperative anatomic measurements. Spine J 14:1895-1901, 2014 
13. Nakashima H, Imagama S, Yukawa Y, Kanemura T, Kamiya M, Yanase M, et al: Multivariate analysis of C-5 palsy incidence after cervical posterior fusion with instrumentation. J Neurosurg Spine 17:103-110, 2012

14. Nassr A, Eck JC, Ponnappan RK, Zanoun RR, Donaldson WF III, Kang JD: The incidence of C5 palsy after multilevel cervical decompression procedures: a review of 750 consecutive cases. Spine (Phila Pa 1976) 37:174-178, 2012

15. Rao R: Neck pain, cervical radiculopathy, and cervical myelopathy: pathophysiology, natural history, and clinical evaluation. Instr Course Lect 52:479-488, 2003

16. Shiozaki T, Otsuka H, Nakata Y, Yokoyama T, Takeuchi K, Ono A, et al: Spinal cord shift on magnetic resonance imaging at 24 hours after cervical laminoplasty. Spine (Phila Pa 1976) 34:274-279, 2009

17. Takeuchi M, Aoyama M, Wakao N, Tawada Y, Kamiya M, Osuka K, et al: Prevalence of C7 level anomalies at the C7 level: an important landmark for cervical nerve ultrasonography. Acta Radiol 57:318-324, 2016

18. Takeuchi M, Kamiya M, Wakao N, Osuka K, Yasuda M, Terasawa T, et al: A simple, 10-minute procedure for transforaminal injection under ultrasonic guidance to effect cervical selective nerve root block. Neurol Med Chir (Tokyo) 54:746-751, 2014

19. Takeuchi M, Wakao N, Hirasawa A, Murotani K, Kamiya $\mathrm{M}$, Osuka K, et al: Ultrasonography has a diagnostic value in the assessment of cervical radiculopathy: a prospective pilot study. Eur Radiol 27:3467-3473, 2017

\section{Disclosures}

This study was funded by the Nagoya Spine Group Foundation. This study received no benefits from any commercial entity either directly or indirectly related to the subject of this manuscript. The authors have reported no conflicts of interest directly relevant to the content of this article.

\section{Author Contributions}

Conception and design: Takeuchi. Acquisition of data: Takeuchi, Wakao, Kamiya, Hirasawa. Analysis and interpretation of data: Takeuchi, Kamiya, Murotani. Drafting the article: Takeuchi, Wakao. Critically revising the article: Takeuchi, Wakao. Reviewed submitted version of manuscript: Takeuchi. Approved the final version of the manuscript on behalf of all authors: Takeuchi. Statistical analysis: Murotani. Administrative/technical/material support: Wakao, Kamiya. Study supervision: Takayasu.

\section{Correspondence}

Mikinobu Takeuchi: Aichi Medical University and Aichi Spine Hospital, Aichi, Japan. neuromicky@hotmail.com. 